with drug treatment, non-adherence to advice by doctors, and the pressures of limited time and resources.

A point in case is the editorial by Joel A Simon on the control of hypertension in elderly people. Clinicians are instructed to treat all patients with a blood pressure of over $160 / 90 \mathrm{~mm} \mathrm{Hg}$, irrespective of age - but not to lower the pressure too far, for fear of entering the upstroke of the J shaped curve. How practical is this advice, and does it lead to demoralisation among those trying to treat their hypertensive patients?

My group practice covers a population of 14500 , is fully computerised, is a research practice of the Royal College of General Practitioners, and runs specific hypertension clinics. How do we measure up to the standards set in the editorial for lowering blood pressure? Of 771 treated patients attending for surveillance of hypertension, a fifth (160) had diastolic pressures of $>90 \mathrm{~mm} \mathrm{Hg}$ and seven tenths (536) had systolic pressures of $>140 \mathrm{~mm} \mathrm{Hg}$. Our practice is unlikely to be much different from many in Britain.

Surely it would be more appropriate for achievable targets to be discussed in an editorial, with health gains per $\mathrm{mm} \mathrm{Hg}$ fall in systolic or diastolic pressure being a more pragmatic (and, indeed, encouraging) goal for primary health care teams genuinely trying their best.

DAVID SEAMARK Honiton Group Practice, Honiton EX14 8DD

1 Simon JA. Treating hypertension: the evidence from clinical trials. $B M \mathcal{F}$ 1996;313:437-8. (24 August.)

\section{Communicating risk}

Use of standard terms is unlikely to result in standard communication

EdrToR,-Kenneth C Calman proposes a language of risk that seeks to introduce standardisation into our communication with patients about risks. ${ }^{1}$ The debate that he calls for must address three fundamental issues. Firstly, is it feasible or theoretically justifiable to translate estimates of risk derived from populations to individuals? Secondly, does current communication about risks meet patients' requirements and is it relevant to them? Thirdly, if we continue with a pragmatic approach to communication about risk, developing it in terms of language, how is this best manifested in practice?

Understanding of risk differs among epidemiologists, clinicians, and lay people. In epidemiology, risk expresses as a statistical measure the association between a characteristic and a disease in a defined population. Tension exists, however, between this perspective, which derives most closely from the "frequentist" interpretation of probability, and the lay perspective. $^{2}$ The lay perspective corresponds with the "subjectivist" interpretation of probability, reflecting the context and our expectations and observations of events. ${ }^{2}$

Clinicians may view themselves as trying to mediate between these two, using epidemiological data about groups to help individual decision making in practice. But communication about clinical risks is not straightforward, which perhaps suggests that estimates of risk derived from populations are unhelpful in the individual setting (as the subjectivist perspective holds) and that current practice in communicating risks fails to meet patients' needs. People often have an "all or nothing" perspective of harm or risk (and may therefore, for example, continue to smoke, or not take any risk with a new drug or an operation), showing that epidemiologically derived estimates of risk are often irrelevant to them. ${ }^{3}$
We still need to know more about what information about risks patients require. Perhaps our language for communicating risks should not be complicated by the uncertainty of applying population estimates to individuals, or perhaps we should continue to bridge this gap from population to individual. This is where a consistent and standardised language could help, but we must address other aspects too. Use of standard terms in the assessment of risk is unlikely to result in standard communication because the interpretation of the language of risks by patients and doctors varies ${ }^{45}$ and because of other contributions to the communication process: non-verbal elements, mental images, past experiences, and discussion about the meaning of risk to individuals. Standard terms would have to be sufficiently flexible to accommodate these contributions. Research to improve communication about risks may need to consider innovation in all these areas and identify whether patient oriented outcome measures are improved by the new processes.

ADRIAN EDWARDS Clinical fellow ROISIN PILI Professor of general practice research NIGEL STOT Professor of general practice University of Wales College of Medicine,

Department of General Practice,

Health Centre,

Maelfa,

Llanedeyrn,

Cardiff CF3 7PN

1 Calman KC. Cancer: science and society and the communication of risk. $B M F$ 1996;313:799-802. (28 September.)

2 Prior L, Bunton R, Nettleton S, Burrows R, eds. Chance and modernity: accidents as a public health problem. In: The sociology of health promotion. London: Routledge, 1995:133 44.

3 Adelsward V, Sachs L. The meaning of 6.8: numeracy and normality in health information talks. Soc Sci Med normality in heal

4 Bryant GD, Norman GR. Expressions of probability: word and numbers. N Engl F Med 1980;302:411.

5 Mazur DJ. Interpretation of graphic data by patients in a general medical clinic. $f$ Gen Intern Med 1990;5:402-5.

Risks can also be categorised as normal or abnormal

EdIToR,-Kenneth C Calman's article on science and society and the communication of risk opens a much needed debate on the public perception of risk. ${ }^{1}$ It defines many of the words used in the assessment of risk, but I would suggest that a further factor that is implicit might be added, though maybe it should be more explicit. To Calman's concept of pairs of terms (avoidable/unavoidable, justifiable/unjustifiable, etc) I would add another pair-normal abnormal. This is close to the acceptable/nonacceptable pair but not identical.

As a community, we accept some risks as normal even when their values are quite high. Examples include accidents on the road ( 1 in 8000), falling off a ladder while painting the bedroom, and cutting off a thumb with a hedge trimmer. These are accepted, albeit with some grumbling, as "part of life." Other risks, such as being infected with a virus in the course of treatment, being exposed to natural radiation, or becoming infected by a virus while swimming, are often perceived as not being part of normal life; though in the range of very low to negligible risks, they are regarded as unacceptable. Some others, such as the risk of being mugged in the street, lie somewhere between and are usually ignored except after a particularly well publicised event.

Whether a particular risk is regarded as normal or abnormal varies greatly over time. For example, we no longer expect a man with a red flag to walk in front of a car, and this reflects how well the general public comes to terms with different examples. Assessment of risks has to go closely with education if we are to manage risk sensibly, and, because it takes time to come to terms with new factors, such education takes longer than we would prefer. Without it, perception is uninformed and the response volatile. As a result, as Calman says, we face spending undue amounts of money on abating negligible risks.

DICK MADELEY

Public Health Laboratory,

Professor of clinical virology

Institute of Pathology,

Newcastle General Hospital,

Newcastle upon Tyne NE4 6BE

1 Calman KC. Cancer: science and society and the communication of risk. $B M Y$ 1996;313:799-802. (28 September.)

\section{Prescribing incentive scheme for non-fundholding general practices}

Tailoring a scheme to individual practices is more effective

EDITOR,-D N Bateman and colleagues' paper adds to the evidence that incentives to general practitioners are an important component of a strategy to change doctors' prescribing patterns. ${ }^{1}$ It is interesting to compare the results of the scheme in the Northern region in England, described by Bateman and colleagues, with those of the rational prescribing payment scheme in Lothian, Scotland. Both schemes contained financial incentives for non-fundholding practices and led to savings in prescribing. The Lothian scheme consisted of three targets for practices: to be within budget, to achieve a rate of prescribing of generic drugs of over $56 \%$, and to achieve a quality target. Table 1-Comparison of main features of schemes in
Northern region and Lothian. Figures are numbers Northern region and Lothian. Figures are
(percentages) except where stated otherwise

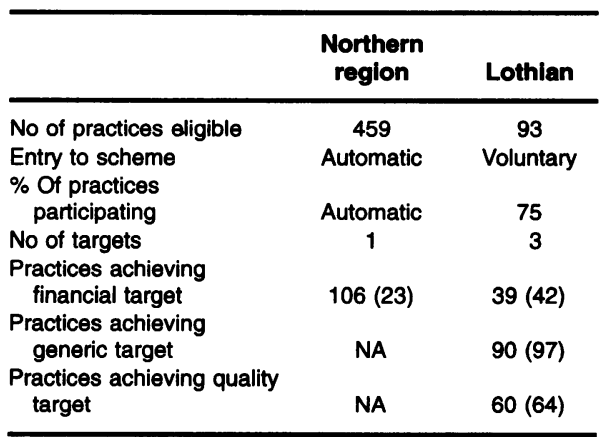

NA $=$ Not applicable

The main difference between the schemes was the way they were applied. In Lothian methods that have been shown to be effective in changing doctors' behaviour were used. These have been described by $\mathrm{Grol}^{2} \mathrm{He}$ recommends the use of face to face education, assessment, and feedback by well respected peers, combined with practical support (including financial stimuli), as a particularly effective means of improving the quality of care. He has shown that coercive controlling methods can have a negative effect at one end of the spectrum and facilitating educational methods a positive effect at the other. An essential part of Lothian's scheme included a practice meeting with the health board's medical prescribing adviser to set the targets and allow discussion.

The success of these schemes could be measured by looking at the number of practices taking part (uptake) or the number achieving their targets, or both. Interestingly, the results are 